Article

\title{
Unique Mitochondrial Single Nucleotide Polymorphisms Demonstrate Resolution Potential to Discriminate Theileria parva Vaccine and Buffalo-Derived Strains
}

\author{
Micky M. Mwamuye ${ }^{1, *} \mathbb{C}$, Isaiah Obara ${ }^{1}$, Khawla Elati ${ }^{1}$, David Odongo ${ }^{2}{ }^{\circledR}$, \\ Mohammed A. Bakheit ${ }^{3}$, Frans Jongejan ${ }^{4}$ and Ard M. Nijhof ${ }^{1, *}$ \\ 1 Institute for Parasitology and Tropical Veterinary Medicine, Freie Universität Berlin, \\ Robert-von-Ostertag-Str. 7-13, 14163 Berlin, Germany; iobara@zedat.fu-berlin.de (I.O.); \\ khawla.elati@fu-berlin.de (K.E.) \\ 2 School of Biological Sciences, University of Nairobi, P.O. Box 30197-00100 Nairobi, Kenya; \\ david.odongo@uonbi.ac.ke \\ 3 Department of Parasitology, Faculty of Veterinary Medicine, University of Khartoum, \\ P.O. Box 321-11115 Khartoum, Sudan; mabakheit@uofk.edu \\ 4 Vectors and Vector-Borne Diseases Research Programme, Department of Veterinary Tropical Diseases, \\ Faculty of Veterinary Science, University of Pretoria, Private Bag X04, 0110 Onderstepoort, South Africa; \\ frans.jongejan@up.ac.za \\ * Correspondence: micky.mwamuye@fu-berlin.de (M.M.M.); ard.nijhof@fu-berlin.de (A.M.N.); \\ Tel.: +49-30-838-62326 (A.M.N.)
}

Received: 16 November 2020; Accepted: 8 December 2020; Published: 8 December 2020

check for updates

\begin{abstract}
Distinct pathogenic and epidemiological features underlie different Theileria parva strains resulting in different clinical manifestations of East Coast Fever and Corridor Disease in susceptible cattle. Unclear delineation of these strains limits the control of these diseases in endemic areas. Hence, an accurate characterization of strains can improve the treatment and prevention approaches as well as investigate their origin. Here, we describe a set of single nucleotide polymorphisms (SNPs) based on 13 near-complete mitogenomes of T. parva strains originating from East and Southern Africa, including the live vaccine stock strains. We identified 11 SNPs that are non-preferentially distributed within the coding and non-coding regions, all of which are synonymous except for two within the cytochrome $b$ gene of buffalo-derived strains. Our analysis ascertains haplotype-specific mutations that segregate the different vaccine and the buffalo-derived strains except T. parva-Muguga and Serengeti-transformed strains suggesting a shared lineage between the latter two vaccine strains. Phylogenetic analyses including the mitogenomes of other Theileria species: T. annulata, T. taurotragi, and T. lestoquardi, with the latter two sequenced in this study for the first time, were congruent with nuclear-encoded genes. Importantly, we describe seven T. parva haplotypes characterized by synonymous SNPs and parsimony-informative characters with the other three transforming species mitogenomes. We anticipate that tracking T. parva mitochondrial haplotypes from this study will provide insight into the parasite's epidemiological dynamics and underpin current control efforts.
\end{abstract}

Keywords: Theileria parva; mitogenomes; haplotypes; SNPs; live vaccine

\section{Introduction}

The protozoan parasite Theileria parva that causes East Coast fever (ECF) and Corridor Disease (CD) is considered among the most debilitating tick-borne pathogens in cattle over its endemic range 
in East, Central, and Southern Africa [1]. In typical ECF symptoms, the disease severity is mainly due to the parasites' ability to transform host lymphocytes [2]. Parasitized lymphocytes proliferate uncontrollably and disseminate the dividing parasite into multiple host tissues. Their accumulation in the lungs triggers severe vasculitis, eventually resulting in respiratory failure with death occurring within three to four weeks of infection [3,4]. With mortalities of up to $100 \%$ in susceptible animals, an estimated one million die per year from an estimated risk population of 28 million cattle mainly belonging to livestock farmers with economically constrained livelihoods [5].

Thus, control of the parasite is urgent to livelihood improvement efforts among resource-poor farmers in sub-Saharan Africa, as highlighted by the World Organization for Animal Health (OIE) [6]. Current control methods include strict tick control measures to curtail pathogen transmission. However, this approach relies heavily on acaricide use, which is unsustainable in the long-run due to acaricide resistance challenges, and toxicity concerns in food and the environment [7]. Anti-theilerial chemotherapy is effective but only with early detection of the disease, which is impractical under field conditions $[8,9]$.

Early observations that cattle acquire long-term immunity when challenged with infected ticks under a long-acting antibiotic treatment opened avenues for the development of an alternative control method based on live parasite stocks, which is called the infection and treatment method (ITM) [10,11]. ITM consists of inoculating cattle with cryopreserved T. parva sporozoites combined with simultaneous treatment with long-acting oxytetracyclines [12]. Early experiments revealed that there were varying cross-reactivities between geographical strains $[13,14]$. Due to this limitation, a cocktail of three immunizing parasite stocks known as the 'Muguga cocktail', comprising Serengeti-transformed, Kiambu 5, and Muguga strains, were combined to achieve broad protection against diverse field isolates [11]. Several other strains have been immunologically profiled to identify an isolate that cross-reacts to diverse field strains in ECF endemic areas. Among the identified strains was a Marikebuni stock isolated from the Kenyan Coast that showed cross-protection against several eastern African strains and, a Boleni strain from Zimbabwe which, apart from a cross-reactivity against Eastern and Central African strains, induced mild infections, hence eliminating the need for antibiotic use in ITM protocol [12].

Historically, ECF is traced to have originated from East Africa and spread southwards, first being reported in present-day Zimbabwe and eventually into South Africa [1]. Yet, it is notable that $T$. parva strains from different geographic regions have varying immunological profiles and epidemiological features. For example, an ability to induce a carrier state in which recovered animals remain infective to ticks has been demonstrated in some strains, enabling transmission between cattle by the vector tick, Rhipicephalus appendiculatus [15-17]. This persistence of vaccine strains raised initial concerns about spreading foreign parasite genotypes into endemic countries free of the vaccine parasite stocks, thereby possibly disrupting endemic stability [18].

By contrast, it is also known that some parasite strains, particularly of African buffalo (Syncerus caffer) origin, induce limited parasitosis and parasitemia, are non-persistent and not efficiently transmissible between cattle hosts [19]. These strains are known to cause a more acute clinical syndrome called Corridor Disease in areas where susceptible cattle are exposed to vector ticks infected on buffalo, which are the primary mammalian carrier hosts [20,21]. Based on its unique clinical presentation, which differs from classical ECF, these particular strains were initially recognized as Theileria parva lawrencei in earlier literature; however, this nomenclature was subsequently abolished with increasing molecular and antigenic data confirming similarities between the two strain populations [22-24]. Further, these data have revealed that cattle transmissible strains are a separately maintained subset population of those found in buffalo, and to differentiate between the two populations, T. parva strains are arbitrarily considered to be either of buffalo or cattle-derived for epidemiological reasons [25].

However, the genetic underpinnings of these strain differences are yet to be fully unraveled, and a precise delineation of the various genotypes is lacking [23]. This is partly because of the parasite's biology, which renders it technically unamenable for genomic studies, especially in obtaining 
pure parasite DNA free from host-DNA contamination [26]. An accurate determination of the origin (buffalo or cattle derived) and geographic spread of strains will help intervention and control efforts. Additionally, accurate characterization of T. parva strains will help to track their frequency and distribution in specific populations, and to characterize breakthroughs in areas of live vaccine field deployments. Further, since T. parva has sexual reproductive phases that are associated with genetic recombination [27], unraveling the parasite genotypes could enhance the understanding of the long-term effects of live vaccine components in the field.

Owing to limited or no recombination, uniparental inheritance patterns and a high substitution rate relative to nuclear genomes, mitochondrial genome studies on related apicomplexan parasites have provided clues of the geographical origin and variants of parasites [28,29]. However, the utility of mitochondrial genomes in T. parva in delimiting the strains and their geographical origin remains unexplored. In this study, we sequenced the mitochondrial genomes of ten T. parva strains, found within the parasite's currently known endemic range, as well as some characterized isolates used as vaccine strains. We also included the mitogenomes of nine other T. parva isolates assembled from their whole-genome data that are publicly available [30]. Further, this study assessed the divergence of T. parva from the closely related host-leukocyte transforming species T. annulata, T. taurotragi and T. lestoquardi with an aim to identify phylogenetically informative mitochondrial characters.

\section{Materials and Methods}

\subsection{Source of Isolates}

The parasite material for the different strains consisted of infected frozen ground-up tick supernatants (GUTS), salivary glands (SG), cattle whole blood, or infected lymphocyte cell lines (Table 1). The GUTS and SG were collected from archived T. parva-infected R. appendiculatus specimens from early live vaccination projects. Parasite DNA from GUTS, SG, and cell culture sample sources was extracted using the NucleoSpin Tissue kit (Macherey-Nagel, Düren, Germany), whereas the NucleoSpin Blood Mini (Macherey-Nagel) was used to extract DNA from blood samples.

Table 1. Parasite material and origin of isolates used in the current study.

\begin{tabular}{ccccc}
\hline Strain/Isolate & Origin & Material Used & Year Created * & Reference \\
\hline T. parva (Serengeti-transformed) & Tanzania & GUTS & 1981 & {$[31]$} \\
T. parva (Boleni) & Zimbabwe & GUTS & 1980 & {$[32]$} \\
T. parva (Pugu I) & Tanzania & Cell culture & 1977 & n.a. \\
T. parva lawrencei (Manyara) & Tanzania & GUTS & 1980 & {$[33]$} \\
T. parva (Satinsyi) & Rwanda & GUTS & 1981 & n.a. \\
T. parva (Kiambu) & Kenya & Salivary glands & 1980 & {$[34]$} \\
T. parva (Marikebuni) & Kenya & GUTS & 1985 & {$[35]$} \\
T. parva (Marula) & Kenya & Blood & 2000 & {$[20]$} \\
T. parva (Muguga) & Kenya & Salivary glands & 1991 & {$[36]$} \\
T. parva (Onderstepoort) & South Africa & GUTS & 1988 & {$[37]$} \\
T. taurotragi & Tanzania & Blood & 2003 & {$[38]$} \\
T. lestoquardi (Atbara) & Sudan & Cell culture & 2001 & {$[39]$} \\
\hline
\end{tabular}

* Indicates the year the material used for this study was created, which may differ from the time when the parasite was first isolated for some strains. n.a.: not available.

\subsection{Next-Generation Sequencing (NGS) T. parva Datasets}

We additionally obtained publicly available whole-genome datasets of nine T. parva strains (DRR002439-46), downloaded in FASTQ from the NCBI (SRA accession number: DRA000613) for assembly of their mitogenomes sequences (Supplementary Table S2). The details of the parasite strains are described in a previous study [30]. All NGS datasets comprised 36 nucleotide, single-end sequence runs performed on the Illumina GAII Analyzer [30]. 


\subsection{Mitogenome Amplification and Sequencing}

Primers were designed based on an alignment of T. parva and T. annulata mitogenomes available in the GenBank (Accession nos. AB499089 and NW_001091933, respectively). A 5808 bp fragment was amplified from all isolated DNA extracts $(0.5-5 \mathrm{ng})$ in $25 \mu \mathrm{L}$ reaction volumes comprising; $0.5 \mathrm{U}$ of S7 Fusion polymerase (Biozym Scientific, Hessisch Oldendorf, Germany), $5 \times$ GC Phusion buffer (ThermoFisher Scientific GmbH, Darmstadt, Germany), $200 \mathrm{mM}$ of dNTPs mix, and $0.5 \mu \mathrm{M}$ of each primer (Supplementary Table S1). The cycling conditions were as follows: $98^{\circ} \mathrm{C}$ for $30 \mathrm{~s}$, followed by 44 cycles of $98^{\circ} \mathrm{C}$ for $15 \mathrm{~s}, 60^{\circ} \mathrm{C}$ for $25 \mathrm{~s}$, and $72{ }^{\circ} \mathrm{C}$ for $4 \mathrm{~min}$. The final extension step was maintained at $72{ }^{\circ} \mathrm{C}$ for $10 \mathrm{~min}$. Amplification of expected $\sim 5.8 \mathrm{~kb}$ fragments was confirmed on $1.5 \%$ agarose gels stained with GRGreen DNA stain (Excellgene, Monthey, Switzerland) under UV trans-illumination. The amplicons were purified using GeneJET PCR Purification Kit (ThermoFisher Scientific GmbH, Darmstadt, Germany) before cloning using the Strataclone blunt vector (Agilent Technologies, USA) under the manufacturer's instructions. The plasmid was purified using the GenUP' ${ }^{\mathrm{TM}}$ Plasmid Kit (biotechrabbit $\mathrm{GmbH}$, Berlin, Germany) and evaluated for targeted inserts based on the EcoRI digestion (ThermoFisher Scientific GmbH, Darmstadt, Germany). The plasmids were sequenced by Sanger technology using standard vector primers (LGC Genomics GmbH, Berlin, Germany) and 10 primers designed in this study to amplify overlapping regions of the mitogenome (Supplementary Table S1).

\subsection{Assembly, Mapping, and Annotation}

The Sanger generated sequences were assembled in Geneious prime 2020.2.3 (www.geneious.com) by creating consensus sequences from the approximately $1000 \mathrm{bp}$ overlapping reads aligned to a reference mitogenome (GenBank Accession: AB499089), which is based on T. parva Muguga vaccine strain. Similarly, the Illumina NGS reads were mapped with reference to (AB499089) using the Geneious mapper under medium-low sensitivity with fine-tuning of at least five iterations. Consensus sequences were generated from contigs based on a threshold of at least $60 \%$ of the adjusted chromatogram quality of contributing bases, while ignoring reads mapped to multiple locations on the reference. The same GenBank reference was used to map and annotate protein-coding genes (PCGs) and the known rRNA genes based on nucleotide similarities.

\subsection{Phylogenetic Analysis and Identification of Informative Single Nucleotide Polymorphisms (SNPs)}

Mitogenome sequence alignments generated using MAFFT [40] as well as concatenated alignments of $\operatorname{cox} 1$ and cob sequences with additional GenBank retrieved sequences of non-transforming Theileria spp. and Babesia spp. were used to infer maximum-likelihood phylogenies. We selected best-fit models for nucleotide substitution based on the lowest Bayesian information (BIC) scores calculated using the jModel Test 2 program and tested nodal support with 100 bootstrap replicates [41]. Phylogenetic trees were generated using PhyML implemented as a plugin within the Geneious software platform [42].

To avoid the challenges of missing data due to incomplete read coverage of the Illumina assemblies, we used only the Sanger data to generate the multiple alignments used for the SNPs detection. We aligned the ten $T$. parva Sanger-generated mitogenome sequences together with three other host-transforming species; T. taurotragi, T. lestoquardi and T. annulata (retrieved from GenBank: NW_001091933). SNPs were identified in Geneious prime with reference to the T. parva Muguga GenBank AB499089 sequence under default settings, with analysis of the variants on protein translations based on Mold-Protozoan Mitochondrial genetic code. We determined informative SNPs for the 13 mitogenomes under the parsimony optimality criterion with equal weights for all characters using PAUP*4.0 software [43]. 
2.6. T. parva Mitogenomes Haplotypes Definition and Network Analysis

Using a modified approach from [30], a second set of SNPs with consideration to the ten T. parva Sanger mitogenomes was extracted from the initial parsimony-informative SNPs. We used DnaSP v.6.12.03 on the second SNP data set to generate T. parva haplotype data [44] and a median-joining (M-J) network was constructed using Network V. 10 software (https://www.fluxus-technology.com/) under default settings to examine relationships among the T. parva mitogenomes [45]. Of the Illumina assembled mitogenomes, strains that had missing data with respect to the second SNP data set were excluded from the haplotype analysis.

\section{Results}

At least ten bidirectional overlapping Sanger reads were obtained for each strain, which were assembled into mitogenomes sequences ranging in size from 5800 to $5811 \mathrm{bp}$. The sequences are archived in NCBI's GenBank under accession numbers MW172707-MW172717; MW218514. The content and gene order for all ten T. parva, one T. taurotragi, and one T. lestoquardi mitogenomes were consistent with previous data, comprising three PCGs, fragmented rRNAs, and no tRNA [46] (Figure 1). 


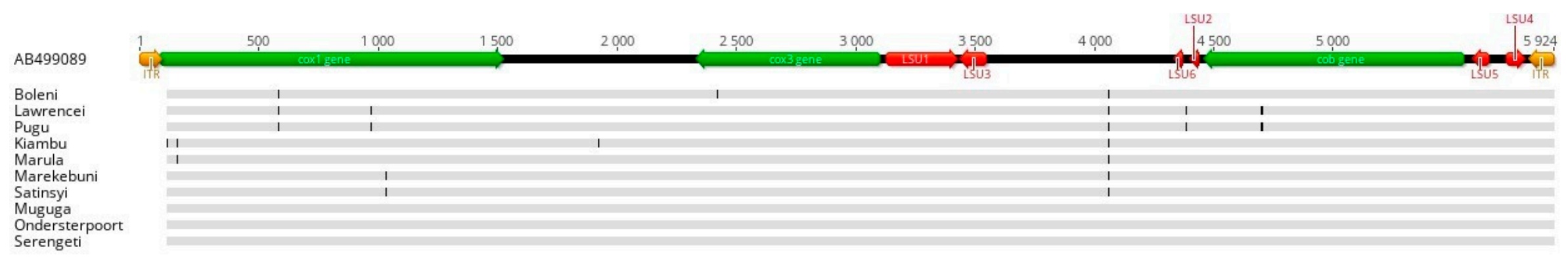

Figure 1. Linear map of T. parva mitochondrial genome and alignment showing the distribution of variants (SNPs) across the mitogenome sequences obtained by Sanger sequencing. cox1 and cox3: cytochrome oxidase subunits; cob: Cytochrome b; LSU: large subunit; ITR: Inverted terminal repeat region; SNPs: single nucleotide polymorphisms; vertical markings indicate polymorphisms in respective nucleotide sequence relative to the reference sequence AB499089 above. 


\subsection{Divergence of T. parva from Other Host-Lymphocyte Transforming Theileria sp.}

Due to length variations, we considered 5793 positions in the multiple alignment of the sanger-sequenced T. parva mitogenomes and the three additional host-lymphocyte transforming Theileria spp. Of the positions considered, there were 42 indels and 1036 SNPs. However, only 662 of the SNPs were parsimony informative across all mitogenome sequences. In terms of percentage identities of the PCG, cob was the most diverse gene, having 73.2-79.6\% identity between T. parva Muguga strain and the three host-lymphocyte transforming Theileria (Figure 2). Phylogenetic analyses were congruent both using the whole mitogenomes and the concatenated gene sequences. In all instances, T. annulata and T. lestoquardi consistently formed an outgroup clade to T. parva and T. taurotragi (Figure 3; Appendix A, Figure A1).

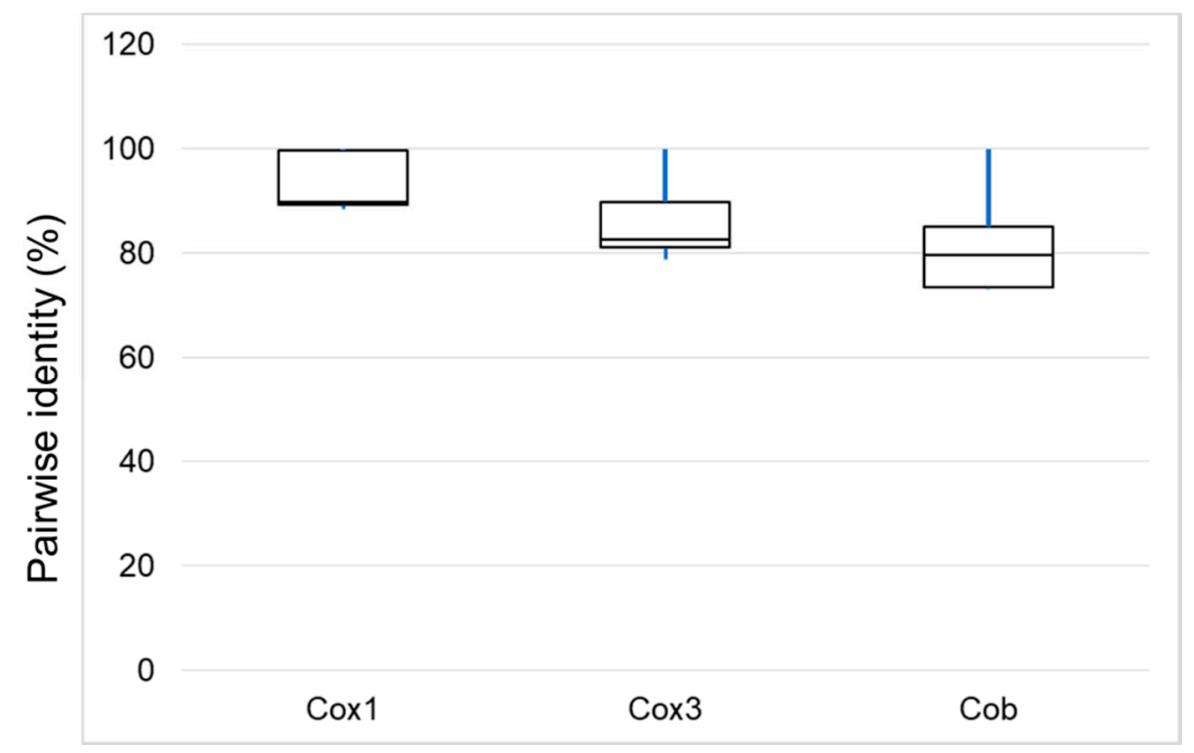

Figure 2. Percentage pairwise identity of the three protein-coding genes across the 14 mitogenomes analyzed in this study. The 25 th and 75 th percentiles are represented by the box limits; lines across the boxes indicate the median; whiskers extend to the maximum and minimum (\%) identity values.

A

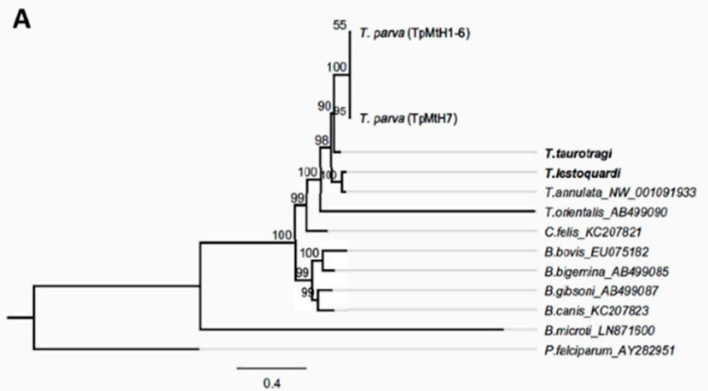

B

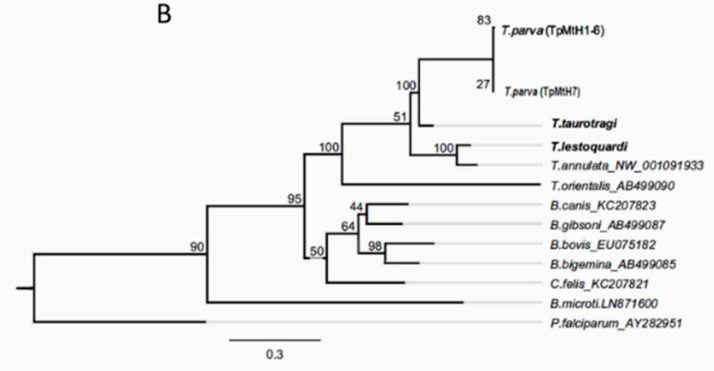

Figure 3. Maximum likelihood phylogeny based on (A) near-complete whole mitogenome sequences $(\sim 5.8 \mathrm{~kb})$ and $(\mathbf{B})$ cob sequences $(\sim 1.1 \mathrm{~kb})$. The nucleotide substitution models for the tree constructions as determined by the lowest Bayesian information (BIC) values were TVM $+G$ and $G T R+G$, respectively. Bootstrap values are based on 100 replicates. Sequences from this study are in bold.

\subsection{T. parva Haplotype Analysis}

We used the extracted second set of SNPs, which comprised nine informative SNPs for T. parva haplotype analysis. As previously noted, we included three Illumina assembled mitogenomes (Nyakizu from Rwanda, MandaliZ22H10, and Buffalo Z5E5 from Zambia) that had data on all the determined nine informative SNPs, irrespective of the other missing regions lacking reads coverage. 
In total, 13 T. parva strains were considered for the haplotype analysis. The SNPs segregated the T. parva strains used into seven haplotypes, which we have identified in this study by assigning the TpmtH prefix numerically beginning with Muguga as a reference sequence (Figure 4). The Muguga strain isolate was assigned into one haplotype identical to Onderstepoort and Serengeti isolates (TpMtH1). Similarly, T. parva lawrencei (Manyara) isolated from an African buffalo, and Pugu I, both from Tanzania, together with a Zambian buffalo isolate (Buffalo Z5E5), formed one haplotype (TpMtH7) that was characterized by two SNPs within both the cox 1 and cob genes. We presumed Pugu I to have originated from buffalo T. parva based on analysis of its sporozoite surface (p67) antigen gene, which showed that it lacked the typical 129 bp deletion that is present in cattle transmissible T. parva [47,48]. The p67 sequence generated (See Appendix A for primer information and cycling conditions) for the Pugu I isolate is archived under accession no MW183674 in the GenBank.

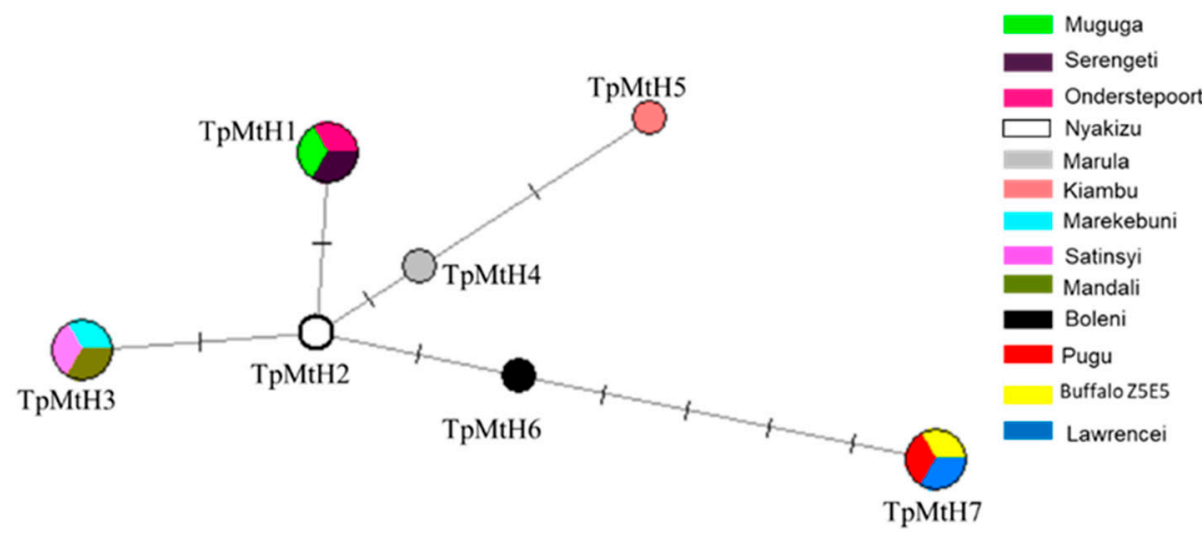

Figure 4. A median-joining (MJ) parsimony network for the $13 \mathrm{~T}$. parva haplotype mitogenome sequences. Node labels TpMtH1-7 represents the unique haplotypes. Lines between nodes indicate mutation points. Larger and fractionated nodes indicate shared haplotypes with multiple strains, each marked with a different color key, as shown.

Further, the Marikebuni strain originating from the Kenyan coast, Mandali from Zambia, and Satinsyi strain from Rwanda formed one haplotype (TpMtH3) characterized by two SNP transitions relative to T. parva Muguga (Table 2; Figure 4). The Marula and Kiambu-V isolates formed independent haplotypes (TpMtH4 and 5), but differed with one SNP position (119) between them (Figure 5). The Boleni isolate (TpMtH6) possessed a transversion mutation within the cox1 gene (SNP 584). This transversion mutation was also notable within the buffalo haplotype (TpMtH7). Interestingly, all haplotypes deviate from TpMtH1 by a transition $(\mathrm{A} \rightarrow \mathrm{G})$ at SNP position 4060 , which lies in a currently functionally unknown region, but appears to be ancestral in the other transforming Theileria (Table 2; Figure 5). This transition is the single defining SNP of the Nyakizu (Rwanda) strain from Muguga, and makes TmMtH2 a central node from which all other haplotypes deviate. However, there was no apparent differentiation by geographic origin as the M-J network nodes associated with multiple haplotypes clustered isolates of diverse origin (Figure 4). 
Table 2. SNPs among the seven haplotypes based on the GenBank reference AB499089. The reference sequence matches haplotype TpMtH1 in the present study.

\begin{tabular}{|c|c|c|c|c|c|c|c|}
\hline Haplotype & Gene & Variant Type & Change & Codon Change & Codon Position & AA Change & $\begin{array}{c}\text { Protein } \\
\text { Effect }\end{array}$ \\
\hline & Cox1 & & & & & & \\
\hline $\mathrm{TpMtH3}$ & & Transition & $\mathrm{C} \rightarrow \mathrm{T}$ & $\mathrm{GCC} \rightarrow \mathrm{GCT}$ & 951 & & \\
\hline TpMtH4 & & Transition & $\mathrm{C} \rightarrow \mathrm{T}$ & $\mathrm{CTG} \rightarrow \mathrm{TTG}$ & 76 & & \\
\hline \multirow[t]{2}{*}{ TpMtH5 } & & Transition & $\mathrm{G} \rightarrow \mathrm{A}$ & GTG $\rightarrow$ GTA & 36 & & \\
\hline & & Transition & $\mathrm{C} \rightarrow \mathrm{T}$ & $\mathrm{CTG} \rightarrow \mathrm{TTG}$ & 76 & & \\
\hline TpMtH6 & & Transversion & $\mathrm{A} \rightarrow \mathrm{C}$ & $\mathrm{GTA} \rightarrow \mathrm{GTC}$ & 501 & & \\
\hline \multirow[t]{2}{*}{ TpMtH7 } & & Transversion & $\mathrm{A} \rightarrow \mathrm{C}$ & $\mathrm{GTA} \rightarrow \mathrm{GTC}$ & 501 & & \\
\hline & & Transition & $\mathrm{C} \rightarrow \mathrm{T}$ & $\mathrm{TAC} \rightarrow \mathrm{TAT}$ & 891 & & \\
\hline TpMtH6 & $\operatorname{Cox3}$ & Transition & $\mathrm{T} \rightarrow \mathrm{C}$ & $\mathrm{CAA} \rightarrow \mathrm{CAG}$ & 555 & & \\
\hline \multirow[t]{2}{*}{ TpMtH7 } & & $\begin{array}{l}\text { Transition } \\
\text { Transition }\end{array}$ & $\begin{array}{l}\mathrm{A} \rightarrow \mathrm{G} \\
\mathrm{A} \rightarrow \mathrm{G}\end{array}$ & $\begin{aligned} \mathrm{GTT} & \rightarrow \mathrm{GCT} \\
\mathrm{GTA} & \rightarrow \mathrm{GCA}\end{aligned}$ & $\begin{array}{l}848 \\
851\end{array}$ & $\begin{array}{l}\mathrm{V} \rightarrow \mathrm{A} \\
\mathrm{V} \rightarrow \mathrm{A}\end{array}$ & $\begin{array}{l}\text { Substitution } \\
\text { Substitution }\end{array}$ \\
\hline & Intergenic & & & & SNP position & & \\
\hline TpMtH2 & & Transition & $\mathrm{A} \rightarrow \mathrm{G}$ & & 4060 & & \\
\hline TpMtH3 & & Transition & $\mathrm{A} \rightarrow \mathrm{G}$ & & 4060 & & \\
\hline TpMtH4 & & Transition & $\mathrm{A} \rightarrow \mathrm{G}$ & & 4060 & & \\
\hline \multirow[t]{2}{*}{ TpMtH5 } & & Transition & $\mathrm{T} \rightarrow \mathrm{C}$ & & 1924 & & \\
\hline & & Transition & $\mathrm{A} \rightarrow \mathrm{G}$ & & 4060 & & \\
\hline TpMtH6 & & Transition & $\mathrm{A} \rightarrow \mathrm{G}$ & & 4060 & & \\
\hline \multirow[t]{2}{*}{ TpMtH7 } & & Transition & $\mathrm{A} \rightarrow \mathrm{G}$ & & 4060 & & \\
\hline & & Transversion & $\mathrm{T} \rightarrow \mathrm{A}$ & & 4382 & & \\
\hline
\end{tabular}

$\begin{array}{lllll}1 & 4 & 4 & 4 & 4\end{array}$

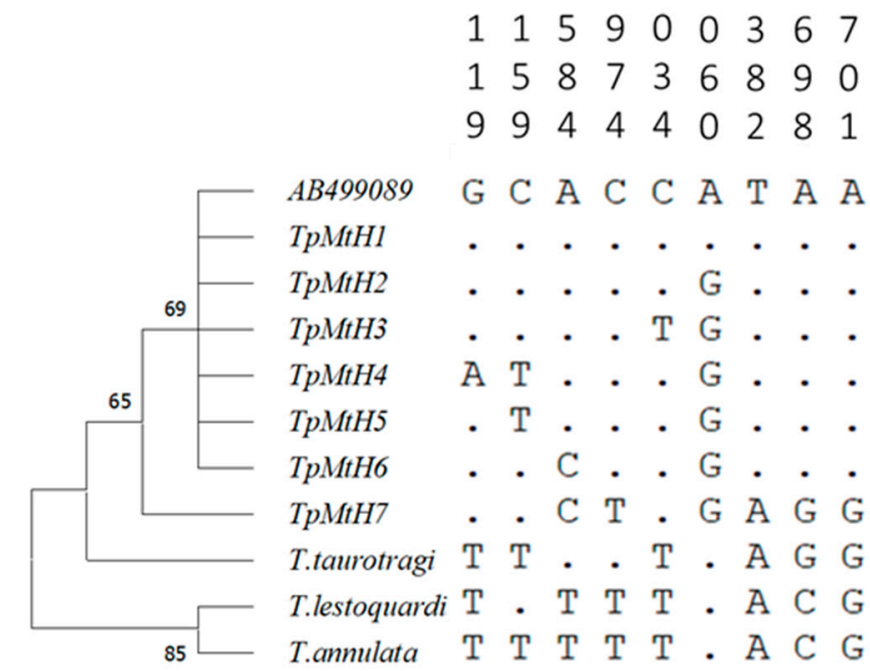

Figure 5. Phylogenetic grouping of the seven T. parva haplotypes identified in this study. The neighbor-joining tree is constructed based on the Jukes-Cantor Model using 9 T. parva only SNPs out of 662 informative SNPs extracted from 13-mitogenome sequences alignment that included; T. taurotragi, T. annulata, and T. lestoquardi. Numbers behind the nodes indicate bootstrap values based on 1000 replicates. The positions are relative to the AB499089 T. parva Muguga mitogenome sequence. Orthologous positions in the three other transforming Theileria are shown for comparison.

\subsection{Intraspecific Divergence among T. parva Strains}

Relative to the GenBank reference AB499089, an alignment of 10 Sanger-sequenced T. parva mitogenomes showed variation at 11 sites, all of which were SNPs with no indels observed (Figure 1). Of the 11 SNPs, only three were found within the intergenic region and involved one transversion within the haplotype associated with buffalo T. parva. In total, there were three transversion SNPs positions, two of which were observed within the cob gene of the buffalo-associated haplotype. Among the genes, cox3 was most conserved with only a single SNPs position within the Boleni mitogenome, 
while the cox 1 gene had five mutated positions, all of which were synonymous. The remaining two SNPs that were found within the cob gene sequences were non-synonymous and resulted in two contiguous amino acid substitutions in their predicted proteins (Table 2). Both substitutions involved the valine codon, which was replaced by an alanine amino acid codon. Notably, these substitutions were only in the haplotype associated with the buffalo T. parva isolates.

\section{Discussion}

In this study, we describe promising mitogenome-based SNPs that demonstrate precision and convenience in characterizing T. parva strains. Previously identified nuclear-based markers mainly based on a panel of mini- and micro-satellites are sometimes biased due to selective amplification of predominant strain clonotypes during passages through cattle and ticks [27,49]. In addition, since the design of the initial markers relied on the genome of T. parva Muguga stock, some markers are possibly biased in detecting diversity within this stock [50]. Although whole-genome SNPs analysis has been demonstrated to have high discriminatory power in typing vaccine strains, it is yet to find field applications [50,51]. Additionally, obtaining pure parasite DNA for whole-genome sequencing, especially for buffalo-derived T. parva is a hurdle due to its biology and may be complicated in the field where the parasite exists as a mixed diverse population $[23,27]$.

Mitochondrial genomes and their individual genes have been extensively used to study phylogeny and applied in species identification and delimitation across broad taxonomic levels [52]. The majority of apicomplexan mitochondrial genomes that have been sequenced to date exhibit an extreme size reduction, containing at most three protein genes (cox1, cox3, and $c o b)$ and fragmented rRNA genes [53]. The extreme mitogenomes size reduction and a faster coalescence make mtDNA attractive to study differentiated T. parva population strains. Indeed, our analysis revealed haplotype-defining SNPs within the T. parva mitogenomes, which are parsimonious with other host-leukocytes transforming Theileria species. Based on median joining (MJ) parsimony analysis, the T. parva mtDNA sequences generated were segregated into an unambiguous network, congruent with the existence of multiple linked lineages. With respect to the T. parva Muguga isolate haplotype, each haplogroup was defined by synonymous nucleotide changes, except for non-synonymous changes leading to amino acid substitutions within the cob of the buffalo-associated strains; T. parva lawrencei, T. parva Buffalo Z5E5, and one field isolate, T. parva Pugu I.

Our analysis identifies T. parva Muguga and Serengeti-transformed as belonging to the same haplotype characterized by nine defining SNPs positions from the strains used in this study. This is not surprising as previous studies have demonstrated a similar monoclonal antibody profile and conservation on their known T. parva antigen coding genes [50,54]. Further, the two strains are strikingly similar at the whole genome level, with only 420 non-synonymous substitutions in Serengeti-transformed relative to the Muguga reference genome reported [50]. These substitutions occur in a paltry 53 genes (out of over 4000 T. parva genes) mainly within polymorphic multicopy gene families and ATP-binding cassette transporter genes located in subtelomeric ends [50]. With the almost similar identity of the two strains, our results, in addition to previous studies, question the necessity of both Muguga and Serengeti-transformed in the trivalent cocktail instead of a divalent cocktail containing either of the two and Kiambu-V. Interestingly, both Muguga and Serengeti-transformed T. parva strains also shared the same haplotype with a historical isolate T. parva Onderstepoort, a laboratory maintained stock isolated in 1937 on the farm Schoonspruit in the Transvaal, South Africa prior to ECF eradication in this country $[1,37,55]$. Earlier analyses on three T. parva antigen proteins; the Polymorphic immunodominant molecule (PIM), sporozoite surface protein (p67), and p104, have shown that these nuclear-encoded antigen genes are, in fact, identical to those of the Muguga parasite $[50,56]$. It is thus conceivable that the ECF-causing strains derive from a common lineage that can be inferred at the mitochondrial genome.

An important finding of this study is the clustering of buffalo-derived T. parva strains under one haplotype (TpMtH7) with the same nine SNPs. It is noteworthy that the buffalo strains used in this 
study originate from two different countries (Zambia and Tanzania), while the field isolate (Pugu I) was isolated during vaccine field trials in Tanzania. And although a Kenyan Buffalo (T. parva LAWR) from the NGS assembly was not included in the haplotype analysis due to missing data on SNP position (159), all its other SNPs positions also matched haplotype (TpMtH7) (data not shown). The buffalo has long been recognized as the natural reservoir of T. parva. The T. parva strains maintained in cattle are considered a subset population from that maintained in buffalo [23,25]. However, there has not been a definitive genetic basis to differentiate what constitutes a buffalo-derived T. parva and a cattle-derived T. parva or whether their designation as a single species is justified $[23,56]$. The available approach of their differentiation based on the p67 alleles only provides a preliminary indication of presumptive exposure of cattle to buffalo T. parva based on alleles-2, 3, and 4, which are considered highly probable to be of buffalo origin in contrast to allele- 1 that is found in cattle transmitted T. parva, but does not necessarily preclude its presence in buffalo [48,49,57]. Our analysis suggests strain defining mitochondrial SNPs that are potential markers for buffalo-derived T. parva lineages.

Noticeably, the Boleni strain formed a separate haplotype (TpMt6) that shared a transversion mutation within the coxI gene with the buffalo haplotype. This strain was isolated from Zimbabwe from a farm that had experienced a severe theileriosis outbreak in January 1978 [31]. Under the now obsolete trinomial nomenclature of T. parva, it was named Theileria parva bovis, which was associated to what was referred to as January disease [58]. The delineation of this strain from our data is thus a significant find as it agrees with the epidemiological distinctions that have been apparent from earlier investigations on theilerioses caused by T. parva. Further, our analysis identifies the Kenyan-Marekebuni, Zambian-Mandali, and Rwandese Satinsyi strains as one haplotype (TpMtH3). Although the shared haplotypes from widely separated regions may suggest a lack of geographical differentiation of the haplotypes, our observations could also be because of a limited sample size as well as through spread by carrier animals.

A high level of interspecies divergence among the transforming Theileria is observed that is characterized by up to 42 indels with respect to T. parva Muguga. However, a limited polymorphism is observed amongst the $13 \mathrm{~T}$. parva mitogenomes analyzed, which is also observed in other apicomplexan species such as Plasmodium falciparum [59]. Of the eleven T. parva SNPs observed, only nine were informative. We modestly suppose this may be convenient compared to whole-genome-based SNPs in which up to $>120,000$ SNPs have been observed in buffalo strains alone [30]. Additionally, we think our approach to defining SNPs that are foremost parsimonious with other leukocyte transforming Theileria provides initial indications on the potential of the identified SNPs to be informative for typing of recently diverged field T. parva strains from common leukocyte transforming ancestor. Nonetheless, further investigation to test the utility of the SNPs is necessary with a larger field population across the T. parva endemic range, especially in wildlife-livestock areas where 'breakthrough' infections against the trivalent live vaccine are known to occur.

The phylogenetic analysis of both the full-length near-complete mitochondrial genomes and the concatenated cox 1 and cob genes place T. parva and T. taurotragi in one clade, consistent with previous analyses using nuclear genes such as the $18 \mathrm{~S}$ RNA gene. The same phylogenetic tree topology is maintained with the sporozoite surface protein gene and its orthologues in respective leukocyte host-transforming species [60]. Thus, the mitogenomes data's observed congruency with nuclear-based data rules out possibilities of inheritance patterns specific to mitochondria in our analysis.

Our data indicate T. annulata and T. lestoquardi form an outgroup clade among the transforming parasites, reflecting an allopatric speciation separation from T. parva and T. taurotragi, and conforms to their currently known demography. Noticeably, T. taurotragi was initially described as a parasite of the eland (Taurotragus oryx) [61], but is also reported to cause infections in cattle in the known endemic range (Eastern, Central, and Southern Africa) of T. parva and its tick vector R. appendiculatus, alongside other tick vectors [38]. As such, co-infections of T. taurotragi and T. parva are, in fact, frequently common [62]. While the pathogenicity of T. taurotragi in cattle is not clearly understood, 
it has been shown to transform a wide range of host cells in in vitro studies [63], and has been associated with cases of cerebral theileriosis (BCT) [38,64].

Similarly sympatric, T. annulata and T. lestoquardi, occur within the same currently known endemic range (N. Africa, S. Asia, and S. Europe) and are transmitted by ticks belonging to Hyalomma genus. Both are important parasites responsible for heavy economic losses and have an intertwined epidemiology that poses interpretation challenges in their overlap in affected countries [65]. Theileria lestoquardi is a parasite of small ungulates and causes malignant ovine theileriosis, while T. annulata causes bovine tropical theileriosis but also co-infects with the former in sheep [65-67].

In conclusion, this study catalogs SNPs based on mitogenomes of characterized T. parva strains and vaccine stocks that can facilitate their tracking in the field. We identify haplotypes defined by SNPs that are initially parsimonious among transforming Theileria; T. parva, T. annulata, T. taurotragi, and T. lestoquardi mitogenomes, the latter two reported herein for the first time. We anticipate that the knowledge of the circulating haplotypes with reference to the live vaccine strains haplotypes will be insightful in characterizing T. parva epidemiology with important implications for control, and have a predictive value on the success of live vaccine deployments besides characterization of breakthrough infections.

Supplementary Materials: The following are available online at http://www.mdpi.com/2075-1729/10/12/334/s1. Table S1: Amplification and sequencing primer sequences, Table S2: Summary of NGS reads (SRA accession number: DRA000613) mapped to T. parva Muguga-mitochondrial sequence (AB499089).

Author Contributions: Conceptualization, M.M.M. and A.M.N.; methodology, M.M.M.; validation, A.M.N., M.M.M., and I.O.; formal analysis, M.M.M.; investigation, M.M.M. and K.E.; resources, A.M.N., M.A.B., D.O., and F.J.; data curation, M.M.M.; writing-original draft preparation, M.M.M.; writing-review and editing, M.M.M., I.O., K.E., D.O., M.A.B., F.J., and A.M.N.; visualization, M.M.M.; supervision, A.M.N. and I.O.; funding acquisition, A.M.N. All authors have read and agreed to the published version of the manuscript.

Funding: The work was supported by the Deutsche Forschungsgemeinschaft (DFG) (Project No. CL166/4-2). MMM was supported by a doctoral scholarship of the Kenyan government through the National Research Fund (NRF) and the Germany Academic Exchange Service (DAAD). AMN received financial support from the Federal Ministry of Education and Research (BMBF) under project number 01KI1720 as part of the 'Research Network Zoonotic Infectious Diseases'.

Acknowledgments: Open Access funding provided by the Freie Universität Berlin.

Conflicts of Interest: The authors declare no conflict of interest.

\section{Appendix A}

The pugu p67 gene was amplified by PCR in a $25 \mu \mathrm{L}$ reaction using published primers [48]. Briefly, the amplification reaction contained; $5 \times$ High-Fidelity (HF) Phusion buffer (ThermoFisher Scientific), $200 \mu \mathrm{M}$ dNTPs (Biozym Scientific GmbH), $0.5 \mu \mathrm{M}$ forward primer (IL 6133: 5'-ACAAACACAATCCCAAGTTC-3'), $0.5 \mu \mathrm{M}$ reverse primers; IL 7922: 5'-CCTTTACTACGTTGGCG-3'), 0.02 U/ $\mu$ L HF Phusion DNA polymerase, sterile PCR-grade water (Carl Roth $\mathrm{GmbH}, \mathrm{Karlsruhe}$ ), and $2 \mu \mathrm{L}$ of template DNA. The amplification cycle was set as follows; $98^{\circ} \mathrm{C}$ for $30 \mathrm{~s}$, followed by 35 cycles of $98^{\circ} \mathrm{C}$ for $30 \mathrm{~s}$, an annealing step at $58^{\circ} \mathrm{C}$ for $40 \mathrm{~s}$, and elongation at $72{ }^{\circ} \mathrm{C}$ for $1 \mathrm{~min}$. The final extension step was $10 \mathrm{~min}$ at $72{ }^{\circ} \mathrm{C}$. The p67 amplicon was purified using GeneJET PCR Purification Kit (ThermoFisher Scientific), and sequenced using Sanger technology (LGC Genomics, Berlin). The obtained sequence was translated and evaluated for the presence/absence of the 43 amino-acid deletions used to categorize cattle and buffalo derived p67 sequences. 


\section{Appendix B}

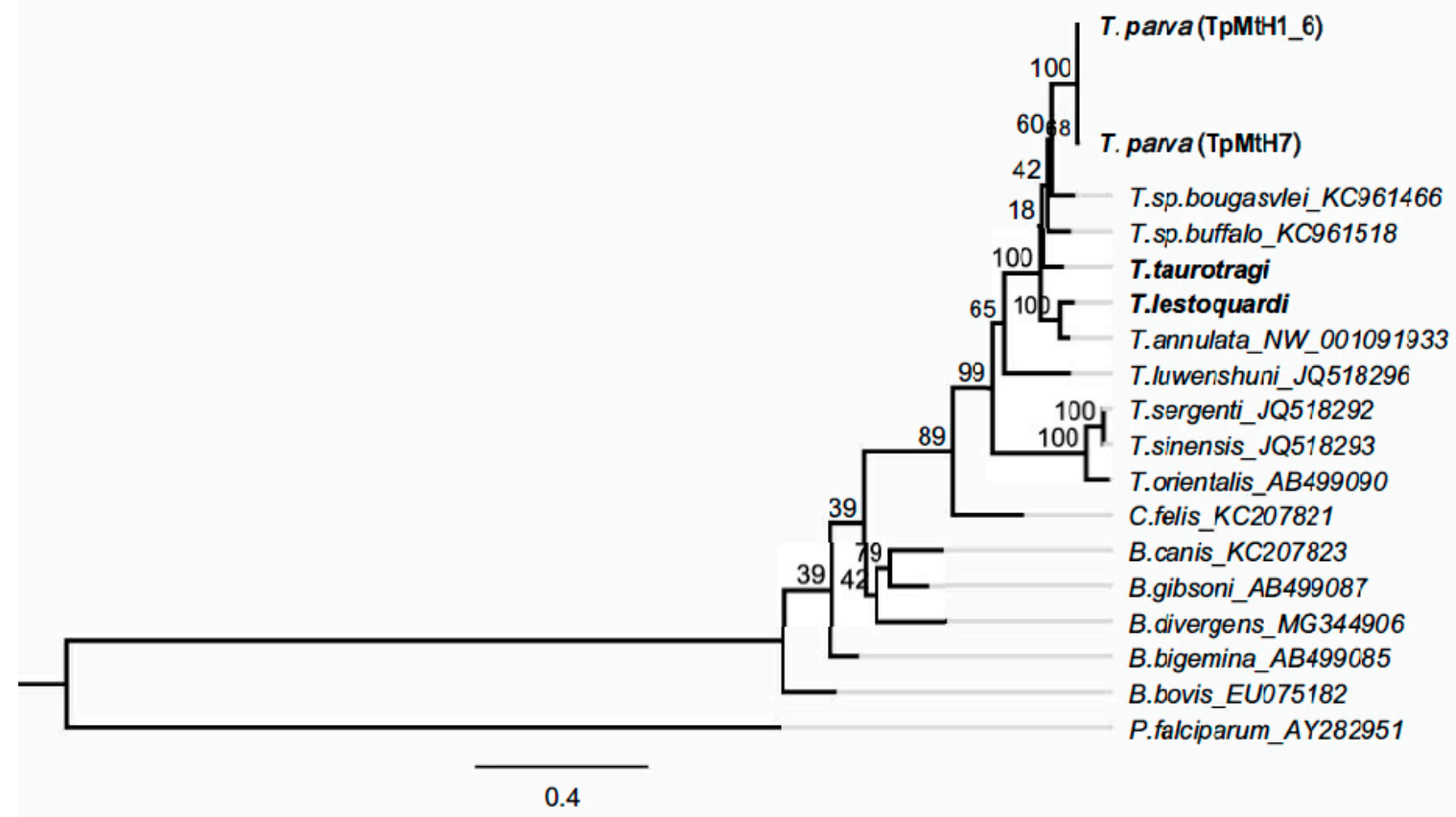

Figure A1. Maximum likelihood phylogeny based on $\operatorname{cox} 1$ gene sequences $(\sim 1.4 \mathrm{~kb})$. The nucleotide substitution model for the tree constructions as determined by the lowest BIC values was GTR + I + G. Bootstrap values are based on 100 replicates. Sequences from this study are in bold.

\section{References}

1. Norval, R.A.I.; Perry, B.D. The Epidemiology of Theileriosis in Africa; Academic Press: London, UK, 1992; pp. 1-41.

2. Tretina, K.; Gotia, H.T. Theileria-transformed bovine leukocytes have cancer hallmarks. Trends Parasitol. 2015, 31, 306-314. [CrossRef]

3. Fry, L.M.; Schneider, D.A. East Coast Fever Caused by Theileria parva is characterized by macrophage activation associated with vasculitis and respiratory failure. PLoS ONE 2016, 11, e0156004. [CrossRef]

4. Irvin, A.D.; Mwamachi, D.M. Clinical and diagnostic features of East Coast Fever (Theileria parva) infection of Cattle. Vet. Rec. 1983, 113, 192-198. [CrossRef]

5. Gachohi, J.; Skilton, R. Epidemiology of East Coast Fever (Theileria parva Infection) in Kenya: Past, present and the future. Parasit. Vectors 2012, 5, 194. [CrossRef]

6. Grace, D.; Songe, M. Impact of neglected diseases on animal productivity and public health in Africa. In Proceedings of the 21st Conference of the OIE Regional Commission for Africa, Rabat, Morocco, 16-20 February 2015.

7. Abbas, R.Z.; Zaman, M.A. Acaricide resistance in cattle ticks and approaches to its Management: The state of play. Vet. Parasitol. 2014, 203, 6-20. [CrossRef]

8. Mbwambo,H.A.; Sudi, F.F. Comparative studies of the efficacy of parvaquone and parvaquone-plus-frusemide in the treatment of Theileria parva infection (East Coast Fever) in cattle. Vet. Parasitol. 2002, 108, 195-205. [CrossRef]

9. D'haese, L.; Penne, K. Economics of theileriosis control in Zambia. Trop. Med. Int. Health 1999, 4, A49-A57. [CrossRef]

10. Neitz, W.O. Aureomycin in Thieileria parva Infection. Nature 1953, 171, 34-35. [CrossRef] [PubMed]

11. Radley, D.E.; Brown, C.G.D. East Coast Fever: 3. Chemoprophylactic immunization of cattle using oxytetracycline and a combination of theilerial strains. Vet. Parasitol. 1975, 1, 51-60. [CrossRef]

12. Morzaria, S.P.; Nene, V. Vaccines against Theileria parva. Ann. N. Y. Acad. Sci. 2006, 916, 464-473. [CrossRef] [PubMed] 
13. Young, A.S.; Brown, C.G.D. Observations on the cross-immunity between Theileria lawrencei (Serengeti) and Theileria parva (Muguga) in Cattle. Int. J. Parasitol. 1973, 3, 723-728. [CrossRef]

14. Cunningham, M.P.; Brown, C.G.D. Theileriosis: The exposure of immunised cattle in a Theileria lawrencei enzootic Area. Trop. Anim. Health Prod. 1974, 6, 39-43. [CrossRef] [PubMed]

15. Young, A.S.; Leitch, B.L. The occurrence of a Theileria parva carrier state in cattle from an East Coast Fever endemic area of Kenya. In Advances in the Control of Theileriosis; Irvin, A.D., Cunningham, M.P., Eds.; Martinus Nijhoff Publishers: The Hague, The Netherlands, 1981; pp. 60-62.

16. Skilton, R.A.; Bishop, R.P. The persistence of Theileria parva infection in cattle immunized using two Stocks which differ in their ability to induce a carrier State: Analysis using a novel blood spot PCR Assay. Parasitology 2002, 124, 265-276. [CrossRef] [PubMed]

17. Olds, C.L.; Mason, K.L. Rhipicephalus appendiculatus ticks transmit Theileria parva from persistently infected cattle in the absence of detectable parasitemia: Implications for East Coast Fever epidemiology. Parasit. Vectors 2018, 11, 126. [CrossRef] [PubMed]

18. Nene, V.; Kiara, H. The Biology of Theileria parva and control of East Coast Fever-Current status and future trends. Ticks Tick. Borne Dis. 2016, 7, 549-564. [CrossRef]

19. Jura, W.G.Z.; Losos, G.J. A Comparative study of the diseases in cattle caused by Theileria lawrencei and Theileria parva. 1. Clinical signs and parasitological observations. Vet. Parasitol. 1980, 7, 275-286. [CrossRef]

20. Bishop, R.P.; Hemmink, J.D. The african buffalo parasite Theileria. sp. (Buffalo) can infect and immortalizecattle leukocytes and encodes divergent orthologues of Theileria parva antigen genes. Int. J. Parasitol. Parasites Wildl. 2015, 4, 333-342. [CrossRef]

21. Young, A.S.; Brown, C.G. Establishment of an experimental field population of Theileria lawrencei-infected ticks maintained by african buffalo (Syncerus caffer). J. Parasitol. 1977, 63, 903-907. [CrossRef]

22. Allsopp, B.A.; Baylish, H.A. Discrimination between six species of Theileria using oligonucleotide probes which detect small subunit ribosomal RNA sequences. Parasitology 1993, 107, 157-165. [CrossRef]

23. Morrison, W.I.; Hemmink, J.D. Theileria parva: A parasite of african buffalo, which has adapted to infect and undergo transmission in cattle. Int. J. Parasitol. 2020, 50, 403-412. [CrossRef]

24. Maritim, A.C.; Young, A.S. Transformation of Theileria parva derived from african buffalo (Syncerus caffer) by tick passage in cattle and its use in infection and treatment immunization. Vet. Parasitol. 1992, 43, 1-14. [CrossRef]

25. Pelle, R.; Graham, S.P. Two Theileria parva CD8 T cell antigen genes are more variable in buffalo than cattle parasites, but differ in pattern of sequence diversity. PLoS ONE 2011, 6, e19015. [CrossRef] [PubMed]

26. Palmateer, N.C.; Tretina, K. Capture-based enrichment of Theileria parva DNA enables full genome assembly of first buffalo-derived strain and reveals exceptional intra-specific genetic diversity. PLOS Negl. Trop. Dis 2020, 14, e0008781. [CrossRef] [PubMed]

27. Katzer, F.; Ngugi, D. Extensive genotypic diversity in a recombining population of the apicomplexan parasite Theileria parva. Infect. Immun. 2006, 74, 5456-5464. [CrossRef]

28. Schmedes, S.E.; Patel, D. Using the Plasmodium mitochondrial genome for classifying mixed-species infections and inferring the geographical origin of P. falciparum parasites imported to the U.S. PLoS ONE 2019, 14, e0215754. [CrossRef]

29. Joy, D.A.; Feng, X. Early origin and recent expansion of Plasmodium falciparum. Science 2003, 300, 318-321. [CrossRef]

30. Hayashida, K.; Abe, T. Whole-Genome Sequencing of Theileria parva strains provides insight into parasite migration and diversification in the African Continent. DNA Res. 2013, 20, 209-220. [CrossRef]

31. Young, A.S.; Purnell, R.E. Transmission of Theileria lawrencei (Serengeti) by the ixodid tick, Rhipicephalus appendiculatus. Trop. Anim. Health Prod. 1973, 5, 146-152. [CrossRef]

32. Lawrence, J.; Mackenzie, P.K. Isolation of a non-pathogenic Theileria of cattle transmitted by Rhipicephalus appendiculatus. Zimbabwe Vet. J. 1980, 11, 27-35.

33. Schreuder, B.E.; Uilenberg, G. studies on Theileriidae (Sporozoa) in Tanzania. VIII. Experiments with african buffalo (Syncerus caffer). Trop. Parasitol. 1977, 28, 367-371.

34. Irvin, A.D.; Purnell, R.E. The application of an indirect method of infecting ticks with piroplasms for use in the isolation of field infections. Br. Vet. J. 1974, 130, 280-287. [CrossRef] 
35. Irvin, A.D.; Dobbelaere, D.A. Immunisation against East Coast Fever: Correlation between monoclonal antibody profiles of Theileria parva stocks and cross immunity in Vivo. Res. Vet. Sci. 1983, 35, 341-346. [CrossRef]

36. Brocklesby, D.W.; Barnett, S.F.; Scott, G.R. Morbidity and mortality rates in East Coast Fever (Theileria parva infection) and their application to drug screening procedures. Br. Vet. J. 1961, 117, 529-531. [CrossRef]

37. Neitz, W.O. Studies on East Coast Fever. S. Afr. Sci. 1948, 1, 133-135.

38. Catalano, D.; Biasibetti, E. “Ormilo Disease” a disorder of zebu cattle in Tanzania: Bovine Cerebral Theileriosis or new protozoan disease? Trop. Anim. Health Prod. 2015, 47, 895-901. [CrossRef]

39. Bakheit, M.A.; Endl, E. Purification of macroschizonts of a sudanese isolate of Theileria lestoquardi (T. lestoquardi [Atbara]). Ann. N. Y. Acad. Sci. 2006, 1081, 453-462. [CrossRef]

40. Katoh, K.; Standley, D.M. MAFFT Multiple sequence alignment software version 7: Improvements in performance and usability. Mol. Biol. Evol. 2013, 30, 772-780. [CrossRef]

41. Darriba, D.; Taboada, G.L. JModelTest 2: More Models, New Heuristics and Parallel Computing. Nat. Methods 2012, 30, 772. [CrossRef]

42. Guindon, S.; Dufayard, J.F. New algorithms and methods to estimate maximum-likelihood phylogenies: Assessing the performance of PhyML 3.0. Syst. Biol. 2010, 59, 307-321. [CrossRef]

43. Swofford, D. PAUP* Phylogenetic Analysis Using Parsimony ( ${ }^{*}$ and Other Methods). Version 4; Sinauer Associates: Sunderland, MA, USA, 2003.

44. Rozas, J.; Ferrer-Mata, A.; Sanchez-DelBarrio, J.C.; Guirao-Rico, S.; Librado, P.; Ramos-Onsins, S.E.; Sanchez-Gracia, A. DnaSP 6: DNA sequence polymorphism analysis of large data sets. Mol. Biol. Evol. 2017, 34, 3299-3302. [CrossRef]

45. Bandelt, H.J.; Forster, P. Median-Joining networks for inferring intraspecific Phylogenies. Mol. Biol. Evol. 1999, 16, 37-48. [CrossRef] [PubMed]

46. Hikosaka, K.; Watanabe, Y. Divergence of the mitochondrial genome structure in the apicomplexan parasites, Babesia and Theileria. Mol. Biol. Evol. 2009, 27, 1107-1116. [CrossRef] [PubMed]

47. Obara, I.; Ulrike, S.; Musoke, T.; Spooner, P.R.; Jabbar, A.; Odongo, D.; Kemp, S.; Silva, J.C.; Bishop, R.P. Molecular evolution of a central region containing B Cell epitopes in the gene encoding the P67 sporozoite antigen within a field population of Theileria parva. Parasitol. Res. 2015, 114, 1729-1737. [CrossRef] [PubMed]

48. Nene, V.; Musoke, A. Conservation of the sporozoite p67 vaccine atigen in cattle-derived Theileria parva stocks with different cross-immunity profiles. Infect. Immun. 1996, 64, 2056-2061. [CrossRef] [PubMed]

49. Oura, C.A.L.; Odongo, D.O. A Panel of microsatellite and minisatellite markers for the characterisation of field isolates of Theileria parva. Int. J. Parasitol. 2003, 33, 1641-1653. [CrossRef]

50. Norling, M.; Bishop, R.P. The genomes of three stocks comprising the most widely utilized live sporozoite Theileria parva vaccine exhibit very different degrees and patterns of sequence divergence. BMC Genom. 2015, 16, 729. [CrossRef]

51. Bishop, R.P.; Odongo, D. A review of recent research on Theileria parva: Implications for the Infection and Ireatment vaccination method for control of East Coast Fever. Transbound. Emerg. Dis. 2020, 67, 56-67. [CrossRef]

52. Bernt, M.; Braband, A. Genetic aspects of mitochondrial genome evolution. Mol. Phylogenet Evol. 2013, 69, $328-338$. [CrossRef]

53. Hikosaka, K.; Kita, K.; Tanabe, K. Diversity of Mitochondrial Genome Structure in the Phylum Apicomplexa. Mol. Biochem. Parasitol. 2013, 188, 26-33. [CrossRef]

54. Bishop, R.; Geysen, D. Molecular and immunological characterisation of Theileria parva stocks which are components of the 'muguga cocktail' used for vaccination against east coast fever in cattle. Vet Parasitol. 2001, 94, 227-237. [CrossRef]

55. Neitz, W.O. Theileriosis, Gonderioses and Cytauxzoonoses: A Review. Onderstepoort J. Vet. Res. 1957, $27,275-326$.

56. Sibeko, K.P.; Collins, N.E. Analyses of genes encoding Theileria parva p104 and Polymorphic Immunodominant Molecule (PIM) reveal evidence of the presence of cattle-type alleles in the South African T. parva Population. Vet. Parasitol. 2011, 181, 120-130. [CrossRef] [PubMed]

57. Sitt, T.; Henson, S. Similar levels of diversity in the gene encoding the p67 sporozoite surface protein of Theileria parva are observed in blood samples from buffalo and cattle naturally infected from buffalo. Vet. Parasitol. 2019, 269, 21-27. [CrossRef] [PubMed] 
58. Uilenberg, G.; Perié, N.M. Causal agents of bovine theileriosis in southern Africa. Trop. Anim. Health Prod. 1982, 14, 127-140. [CrossRef] [PubMed]

59. Preston, M.D.; Campino, S. A barcode of organellar genome polymorphisms identifies the Geographic Origin of Plasmodium falciparum strains. Nat. Commun. 2014, 5, 4052. [CrossRef]

60. Sivakumar, T.; Hayashida, K. Evolution and genetic diversity of Theileria. Infect. Genet. Evol. 2014, 27, $250-256$. [CrossRef]

61. Brocklesby, D.W.; Martin, H. A new parasite of the eland. Vet. Rec. 1960, 72, 331-332.

62. Njiiri, N.E.; de Bronsvoort, B.M.C. The epidemiology of tick-borne haemoparasites as determined by the Reverse Line Blot Hybridization assay in an intensively studied cohort of calves in western Kenya. Vet. Parasitol. 2015, 210, 69-76. [CrossRef]

63. Stagg, D.A.; Young, A.S. Infection of mammalian cells with Theileria Species. Parasitology 1983, 86, $243-254$. [CrossRef]

64. Biasibetti, E.; Sferra, C.; Lynen, G.; Di Giulio, G.; De Meneghi, D.; Tomassone, L.; Valenza, F.; Capucchio, M.T. Severe Meningeal Fibrinoid Vasculitis Associated with Theileria taurotragi Infection in Two Short-Horned Zebu Cattle. Trop. Anim. Health Prod. 2016, 48, 1297-1299. [CrossRef]

65. Brown, C.G.D.; Ilhan, T. Theileria lestoquardi and T. annulata in cattle, sheep, and goats: In vitro and in vivo Studies ${ }^{a}$. Ann. N. Y. Acad. Sci. 1998, 849, 44-51. [PubMed]

66. Al-Hamidhi, S.; Weir, W. Theileria lestoquardi displays reduced genetic diversity relative to sympatric Theileria annulata in Oman. Infect. Genet. Evol. 2016, 43, 297-306. [CrossRef] [PubMed]

67. Bishop, R.; Musoke, A. Theileria: Intracellular Protozoan Parasites of Wild and Domestic Ruminants Transmitted by Ixodid Ticks. Parasitology 2004, 129, S271-S283. [CrossRef] [PubMed]

Publisher's Note: MDPI stays neutral with regard to jurisdictional claims in published maps and institutional affiliations. 